Research article Open Access

\title{
Mycophenolate sodium treatment in patients with primary Sjögren syndrome: a pilot trial
}

\author{
Peter Willeke1, Bernhard Schlüter ${ }^{2}$, Heidemarie Becker ${ }^{1}$, Heiko Schotte ${ }^{1}$, Wolfram Domschke ${ }^{1}$ \\ and Markus Gaubitz ${ }^{1}$
}

\author{
1Department of Medicine B, Muenster University Hospital, Albert Schweitzer Street 33, D-48129 Muenster, Germany \\ ${ }^{2}$ Institute of Clinical Chemistry and Laboratory Medicine, Muenster University Hospital, Albert Schweitzer Street 33, D-48129 Muenster, Germany \\ Corresponding author: Peter Willeke, willeke.peter@uni-muenster.de \\ Received: 20 Jul 2007 Revisions requested: 11 Sep 2007 Revisions received: 24 Sep 2007 Accepted: 6 Nov 2007 Published: 6 Nov 2007 \\ Arthritis Research \& Therapy 2007, 9:R115 (doi:10.1186/ar2322) \\ This article is online at: http://arthritis-research.com/content/9/6/R115 \\ (c) 2007 Willeke et al.; licensee BioMed Central Ltd. \\ This is an open access article distributed under the terms of the Creative Commons Attribution License (http://creativecommons.org/licenses/by/2.0), \\ which permits unrestricted use, distribution, and reproduction in any medium, provided the original work is properly cited.
}

\begin{abstract}
The aim of this study was to evaluate the efficacy and safety of mycophenolate sodium (MPS) in patients with primary Sjögren syndrome (pSS) refractory to other immunosuppressive agents. Eleven patients with pSS were treated with MPS up to 1,440 $\mathrm{mg}$ daily for an observation period of 6 months in this singlecenter, open-label pilot trial. At baseline, after 3 months, and after 6 months, we examined the clinical status, including glandular function tests, as well as different laboratory parameters associated with pSS. In addition, subjective parameters were determined on the basis of different questionnaires. Treatment with MPS was well tolerated in 8 of 11 patients. Due to vertigo or gastrointestinal discomfort, two patients did not complete the trial. One patient developed pneumonia 2 weeks after treatment and was withdrawn. In the

remaining patients, MPS treatment resulted in subjective improvement of ocular dryness on a visual analogue scale and a reduced demand for artificial tear supplementations. However, no significant alterations of objective parameters for dryness of eyes and mouth were observed, although a substantial improvement of glandular functions occurred in two patients with short disease duration. In addition, treatment with MPS resulted in significant reduction of hypergammaglobulinemia and rheumatoid factors as well as an increase of complement levels and white blood cells. MPS promises to be an additional therapeutic option for patients with pSS, at least in those with shorter disease duration. Further investigations about the efficacy and safety of MPS in pSS have to be performed in larger numbers of patients.
\end{abstract}

\section{Introduction}

Primary Sjögren syndrome (pSS) is an autoimmune disorder characterized by keratoconjunctivitis sicca and xerostomia. In addition, various extraglandular manifestations may develop. Several immunomodulating agents have been attempted in the treatment of pSS without achieving satisfactory results [1]. Currently, there is no approved systemic treatment for pSS.

Mycophenolic acid (MPA) is a selective inhibitor of inosine monophosphate dehydrogenase which leads to inhibition of the de novo pathway of nucleotide synthesis. The antiproliferative effect of MPA mainly affects activated T and B lymphocytes because the proliferation of these cells is critically dependent on the de novo purine synthesis compared with other eukaryotic cells [2]. Since these lymphocytes have been suggested to play a pivotal role in the inflammation and immunopathogenesis of pSS $[3,4]$. ., MPA might be a promising agent in the treatment of pSS.

MPA-containing compounds such as mycophenolate mofetil (MMF) and enteric-coated mycophenolate sodium (MPS) are immunosuppressive drugs approved for the prevention of transplant rejection [5]. MPS $720 \mathrm{mg}$ and MMF $1,000 \mathrm{mg}$ deliver nearly equimolar doses of the active immunosuppressive agent [6].

MMF is an effective treatment in systemic lupus erythematosus (SLE) $[7,8]$. and other autoimmune diseases $[9,10]$. MMF has

$\overline{\mathrm{AE}}=$ adverse event; $\mathrm{ESR}=$ erythrocyte sedimentation rate; $\mathrm{Gl}=$ gastrointestinal; $\mathrm{Ig}=$ immunoglobulin; $\mathrm{MMF}=$ mycophenolate mofetil; MPA $=$ mycophenolic acid; MPS = mycophenolate sodium; pSS = primary Sjögren syndrome; RF = rheumatoid factor; SF-36= Short Form 36; SLE = systemic lupus erythematosus; VAS $=$ visual analogue scale. 
been used as maintenance therapy after treatment with rituximab (anti-CD20 antibody) in a patient with pSS [11]. We reported a case of successful treatment with MMF in pSS with vasculitis [12]. The recent observations and the immunosuppressive effect of MPA in other autoimmune diseases led us to evaluate the efficacy and safety of MPA treatment in patients with pSS refractory to other immunosuppressive agents.

\section{Materials and methods Study design}

We performed a prospective, single-center, open-label pilot trial for an observation period of 6 months. Medical treatment was initiated with one tablet of $360 \mathrm{mg}$ MPS per day. The dosage was increased weekly by $360 \mathrm{mg}$ up to a maximum stable dose of $1,440 \mathrm{mg}$ daily. In patients not tolerating the drug well, the dosage was reduced to $720 \mathrm{mg}$ per day. All patients gave written informed consent to participate. The study protocol was approved by the local independent ethics committee.

\section{Patient selection criteria}

Inclusion and exclusion criteria for the trial are presented in Table 1. Eligibility criteria included the diagnosis of pSS based on the American-European Consensus criteria [13] provided that the patients had evidence of active disease. Since there are no validated disease activity criteria for pSS, active disease was defined by elevated erythrocyte sedimentation rate (ESR) (>25 mm/hour), hypergammaglobulinemia $(>1,500$ $\mathrm{mg} / \mathrm{dL}$ ) and the presence of autoantibodies (that is, anti-SSA and/or SSB antibodies and/or rheumatoid factor [RF]).

\section{Outcome measures}

Clinical visits were performed at baseline, week 12, and week 24. After 4 weeks, an additional visit, including clinical examination and laboratory tests, was performed. Patients were asked about possible adverse events (AEs) and about the daily demand for artificial teardrops. Clinical assessment consisted of a general physical examination, the 28-joint count of tender/swollen joints, and a tender point count (maximum of 18).

\section{Functional parameters}

The lachrymal gland function was assessed by unanesthetized Schirmer's test [14]. A value of less than $5 \mathrm{~mm}$ per 5 minutes was taken as abnormal. In addition, we collected the unstimulated whole saliva throughout a 5-minute period by performing the spitting technique [15]. A flow rate of less than 0.5 grams per 5 minutes was considered as glandular hypofunction.

\section{Subjective parameters}

Patients were instructed to express the severity of ocular dryness, arthralgia, and fatigue on a $100-\mathrm{mm}$ visual analogue scale (VAS) ranging from 0 for no symptoms to 100 for extreme symptoms. Outcome was also determined by the Short Form 36 (SF-36) questionnaire in eight scales (physical functioning, role physical, bodily pain, general health, vitality, social functioning, role emotional, and mental health) ranging from 0 to 100 [16]. A higher value indicates a higher state of well-being. Aggregated physical component summary score and mental component summary score of the SF-36 were calculated. The SF-36 has been validated for patients with pSS

Table 1

\section{Inclusion and exclusion criteria}

Inclusion criteria Diagnosis of primary Sjögren syndrome based on the American-European Consensus criteria [13]

Erythrocyte sedimentation rate of greater than $25 \mathrm{~mm} /$ hour and hypergammaglobulinemia $(>1,500 \mathrm{mg} / \mathrm{dL})$

Presence of anti-SSA and/or SSB antibodies and/or rheumatoid factor

Requirement of artificial teardrops due to symptomatic sicca syndrome

Inadequate response or intolerance of prior treatment with hydroxychloroquine and/or azathioprine

Adequate contraception for females of childbearing potential

Exclusion criteria Age below 18 or above 75 years

Secondary Sjögren syndrome

History of cancer, severe infections, or other uncontrolled diseases

Treatment with concomitant disease-modifying antirheumatic drugs within the last 8 weeks before baseline evaluation

Prednisolone dose of greater than $5 \mathrm{mg} /$ day or changes of prednisolone dose within the last 4 weeks before baseline

Use of secretagogues (for example, pilocarpine and civemeline) or medications that potentially diminish exocrine gland function (for example, tricyclic antidepressants and anticholinergic drugs)

Pregnant or lactating women 
[17]. In addition, the Health Assessment Questionnaire was completed by our patients [18]. Values range from 0 to 3 . A lower score indicates better health.

\section{Laboratory parameters}

Routine laboratory parameters (that is, ESR, C-reactive protein, renal and liver function tests, total protein, and full blood count) were determined at each visit. Levels of immunoglobulins ( $\lg G, \lg M$, and $\lg \mathrm{A}), \lg M-R F$, and serum concentrations of complement levels (C3 and $\mathrm{C} 4$ ) were measured by nephelometry (BN2; Dade Behring, now Siemens Medical Solutions Diagnostics $\mathrm{GmbH}$, Bad Nauheim, Germany). IgG antibodies to SSA and SSB were analyzed by fluorescence enzyme immunoassay (Phadia GmbH, Freiburg, Germany). Protein electrophoresis was performed on an Olympus Hite 320 (Olympus-Diagnostika GmbH, Rees, Germany).

\section{Statistical analysis}

Data were analyzed by means of the statistic software package SPSS 12.0 (SPSS Inc., Chicago, IL, USA). The significance of changes from baseline was measured by Wilcoxon test. A $p$ value of less than 0.05 was considered significant.

\section{Results \\ Clinical characteristics of patients}

Eleven patients with active pSS were included in this study (Table 2). All patients were women with a mean age of $50.1 \pm$ 10.8 years and a mean disease duration of $9.5 \pm 5.4$ years. All patients had an abnormal Schirmer's test. In six patients, a diminished salivary flow rate was detected. Seven patients had mild leukopenia $(<4,000 / \mu \mathrm{L})$ at baseline evaluation (Table 2$)$. As for other extraglandular manifestations, eight patients had arthralgia, five had Raynaud syndrome, one patient had polyneuropathy, and one had vasculitis.

\section{Safety of mycophenolate sodium}

Eight patients completed the study period of 24 weeks. Two patients were unwilling to continue the study after week 4 due to vertigo (patient 1) and gastrointestinal (Gl) complaints (patient 3). Patient 6 was withdrawn at day 15 after developing pneumonia that caused hospitalization. The patient fully recovered after antibiotic treatment. This event was the only serious $\mathrm{AE}$. The reported AEs possibly related to the study medication were of mild intensity. Gl discomfort (defined by the occurrence of nausea, dyspepsia, or diarrhea) was the most frequent $A E$ in 4 of the 11 patients included (Table 2). These patients had no previous evidence of intestinal involvement associated with the disease. In one patient, a herpes labialis infection occurred and was controlled by local application of aciclovir. Two patients during the study developed a common cold that required no additional medical treatment. MPS was reduced in patients 5 and 10 after week 12 due to mild Gl discomfort and palpitation, respectively, which resolved after a dose reduction of MPS. We observed no significant changes in body weight, blood pressure, or heart rate. Also, no drugrelated hematological abnormalities were observed.

\section{Changes in outcome parameters}

Outcome parameters were evaluated for the eight patients who completed the study (Table 3). No significant changes in the Schirmer's test or the amount of unstimulated whole saliva in the cohort were observed. However, in patients 8 and 11, who had a relatively short disease duration, a significant improvement was observed in both the Schirmer's test $(1.25$

Table 2

\begin{tabular}{|c|c|c|c|c|c|c|c|}
\hline Patient & Age in years & Gender & $\begin{array}{l}\text { Disease duration } \\
\text { in years }\end{array}$ & $\begin{array}{c}\text { Anti-SSA/Anti- } \\
\text { SSB/RF }\end{array}$ & $\begin{array}{l}\text { Extraglandular } \\
\text { manifestations }\end{array}$ & $\begin{array}{l}\text { Prednisolone } \\
\text { (mg/day) }\end{array}$ & Adverse events \\
\hline $1^{\mathrm{a}}$ & 59 & Female & 13 & $+/+/+$ & Arthralgia & - & Vertigo, perspiration \\
\hline 2 & 46 & Female & 12 & $+/+/+$ & $\mathrm{LP}, \mathrm{RS}$ & - & Gl discomfort, common cold \\
\hline $3^{a}$ & 50 & Female & 15 & $+/+/+$ & $\begin{array}{l}\text { Arthralgia, RS, } \\
\text { LP }\end{array}$ & - & Gl discomfort \\
\hline 4 & 67 & Female & 17 & $+/+/+$ & Arthralgia, RS & 2.5 & None \\
\hline 5 & 60 & Female & 4 & $+/+/+$ & $\begin{array}{l}\text { Arthralgia, LP, } \\
\text { VA }\end{array}$ & - & Gl discomfort ${ }^{b}$ \\
\hline $6^{a}$ & 61 & Female & 5 & $+/+/+$ & Arthralgia, LP & - & Pneumonia \\
\hline 7 & 53 & Female & 13 & $+/-/+$ & $\mathrm{LP}, \mathrm{PNP}$ & 2.5 & Gl discomfort, herpes labialis \\
\hline 8 & 40 & Female & 2 & $+/-/-$ & Arthralgia, LP & - & None \\
\hline 9 & 35 & Female & 12 & $+/-/+$ & $\mathrm{LP}, \mathrm{RS}$ & 5 & None \\
\hline 10 & 44 & Female & 9 & $+/+/+$ & Arthralgia, RS & - & Palpitation, perspiration ${ }^{b}$ \\
\hline 11 & 36 & Female & 2 & $+/+/-$ & Arthralgia & - & Common cold \\
\hline
\end{tabular}

aPatients 1, 3, and 6 did not complete the study; bdose was reduced to $720 \mathrm{mg} /$ day after week 12. Gl, gastrointestinal; LP, leukopenia; PNP, polyneuropathy; RF, rheumatoid factor; RS, Raynaud syndrome; VA, vasculitis. 
Table 3

\begin{tabular}{|c|c|c|c|}
\hline Parameter & Baseline & Week 12 & Week 24 \\
\hline \multicolumn{4}{|l|}{ Glandular function tests } \\
\hline Schirmer's test (millimeters per 5 minutes) & $2.0 \pm 3.2$ & $3.4 \pm 3.5$ & $4.4 \pm 4.9$ \\
\hline Whole saliva (grams per 5 minutes) & $0.49 \pm 0.41$ & $0.48 \pm 0.41$ & $0.56 \pm 0.41$ \\
\hline \multicolumn{4}{|l|}{ Laboratory tests } \\
\hline Erythrocyte sedimentation rate (mm/hour) & $47.6 \pm 20.5$ & $44.1 \pm 23.3$ & $44.2 \pm 21.0$ \\
\hline Gamma globulins (g/L) & $21.3 \pm 6.3$ & $19.5 \pm 8.6^{\mathrm{a}}$ & $18.8 \pm 7.5^{b}$ \\
\hline $\lg G(\mathrm{mg} / \mathrm{dL})$ & $2,159 \pm 949$ & $2,048 \pm 755$ & $2,025 \pm 895$ \\
\hline $\lg M(\mathrm{mg} / \mathrm{dL})$ & $189 \pm 108$ & $144 \pm 62.7^{a}$ & $142 \pm 62.8^{a}$ \\
\hline $\lg \mathrm{A}(\mathrm{mg} / \mathrm{dL})$ & $399 \pm 417$ & $359 \pm 363$ & $329 \pm 305$ \\
\hline Rheumatoid factor $\lg \mathrm{M}(\mathrm{IU} / \mathrm{mL})$ & $275 \pm 504$ & $193 \pm 380$ & $179 \pm 317^{a}$ \\
\hline Anti-SSA antibodies $(\mathrm{U} / \mathrm{mL})$ & $5,863 \pm 5,321$ & $6,308 \pm 4,008$ & $5,892 \pm 8,859$ \\
\hline Anti-SSB antibodies $(\mathrm{U} / \mathrm{mL})$ & $1,217 \pm 1,386$ & $1,424 \pm 1,925$ & $1,320 \pm 1,668$ \\
\hline $\mathrm{C} 3(\mathrm{mg} / \mathrm{dL})$ & $99.3 \pm 30.2$ & $101 \pm 33.5$ & $108 \pm 34.7 \mathrm{~b}$ \\
\hline $\mathrm{C} 4(\mathrm{mg} / \mathrm{dL})$ & $14.9 \pm 8.8$ & $16.4 \pm 9.5^{a}$ & $16.8 \pm 8.9^{b}$ \\
\hline \multicolumn{4}{|l|}{ Subjective findings } \\
\hline VAS sicca syndrome (0 to $100 \mathrm{~mm}$ ) & $68.7 \pm 15.6$ & $56.4 \pm 19.9$ & $52.8 \pm 20.8^{a}$ \\
\hline VAS arthralgia (0 to $100 \mathrm{~mm}$ ) & $62.1 \pm 23.4$ & $45.1 \pm 34.3$ & $47.5 \pm 30.0$ \\
\hline VAS fatigue ( 0 to $100 \mathrm{~mm}$ ) & $63.4 \pm 24.0$ & $57.4 \pm 33.4$ & $65.3 \pm 22.6$ \\
\hline Use of artificial teardrops (times per day) & $3.7 \pm 2.3$ & $1.8 \pm 1.3^{a}$ & $1.6 \pm 1.9^{b}$ \\
\hline Health Assessment Questionnaire score & $0.73 \pm 0.77$ & $0.81 \pm 0.84$ & $0.73 \pm 0.80$ \\
\hline
\end{tabular}

a $p<0.05$ versus baseline value analyzed by Wilcoxon signed rank test; ${ }^{b} p<0.02$ versus baseline value analyzed by Wilcoxon signed rank test. Table presents outcome parameters in the eight patients with primary Sjögren syndrome who completed the study. Data are presented as mean \pm standard deviation. Ig, immunoglobulin; VAS, visual analogue scale.

$\pm 0.35 \mathrm{~mm}$ at baseline versus $6.75 \pm 5.3 \mathrm{~mm}$ after 24 weeks) and the unstimulated whole saliva $(0.17 \pm 0.22$ grams per 5 minutes at baseline versus $0.44 \pm 0.11$ grams per 5 minutes at week 24).

No changes in the 28-swollen/tender joint count or in the number of tender points were observed (data not shown). In one patient with vasculitis, a remarkable improvement of the vasculitis of the lower arms and legs was observed after 12 weeks (patient 5). This improvement lasted throughout the study. No significant changes concerning the Raynaud syndrome were observed, although an angiologic examination was not performed as follow-up procedure.

\section{Subjective parameters}

Significant improvement in patients' assessment of ocular dryness on a VAS was observed $(p<0.05)$. The demand for daily artificial tear supplementation decreased significantly during treatment $(p<0.02)$. No significant improvement in the other VAS was observed.
Patients had significant improvement in the general health and the role emotional domains of the SF-36 (Figure 1). Other domains and the physical component summary score did not improve significantly. Although a clear tendency toward improvement of the mental component summary score was observed, statistical significance was not reached $(p=0.06)$.

\section{Laboratory parameters}

We detected a significant reduction of gamma globulins after 12 and 24 weeks of treatment with MPS ( $p<0.05)$. Also, a significant reduction of $\operatorname{lgM}$ was observed after 12 and 24 weeks, whereas the reduction of $\lg G$ or $\lg A$ was not significant. No significant change in ESR was measured. A significant increase of both $\mathrm{C} 3$ and $\mathrm{C} 4$ complement levels occurred during the treatment. We further detected a decrease of IgMRF after 24 weeks. The white blood cell count increased significantly from $4,478 \pm 1,190$ cells per microliter at baseline to $5,703 \pm 1,508$ cells per microliter after 24 weeks $(p<0.05)$ (data not shown). No differences were found in red blood count, thrombocytes, or renal or liver function parameters. 


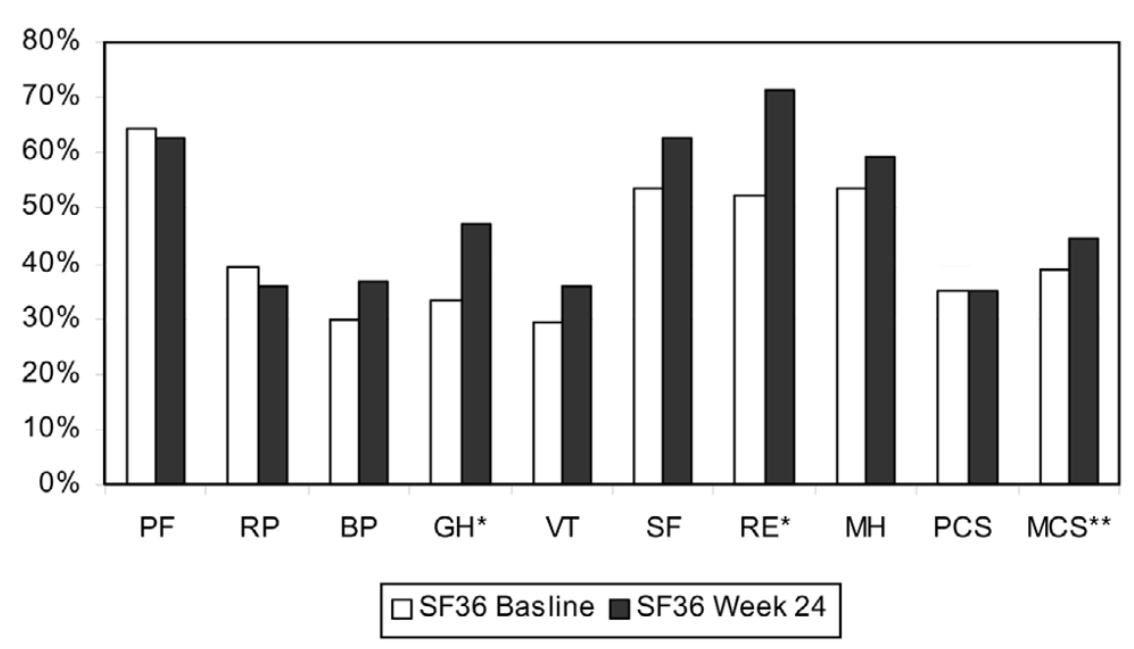

Short Form 36 (SF-36) at baseline and after 24 weeks of treatment with mycophenolate sodium in patients with primary Sjögren syndrome $(n=8)$. The SF-36 domains are physical functioning (PF), role physical (RP), bodily pain (BP), general health (GH), vitality (VT), social functioning (SF), role emotional (RE), mental health (MH), and the physical and mental component summary scores (PCS and MCS). The GH and RE domains increased significantly $(p<0.05)$ after 24 weeks $(*)$. The increase of the MCS did not reach significance $\left({ }^{*} p=0.06\right)$.

\section{Discussion}

We present the first controlled pilot trial of MPS treatment in patients with pSS. Our study shows that MPS can improve symptoms and laboratory findings in patients with active pSS. The optimum systemic treatment of pSS is still unclear. Although no controlled study has been performed so far, MPA was suggested as sole or adjuvant treatment for pSS in a recent review [19].

We found a significant reduction of ocular dryness assessed by a VAS. In accordance with this finding, the daily demand for artificial teardrops decreased significantly in our patients. On the other hand, no improvement in the salivary and lachrymal gland functions in our cohort was observed. It should be noted, however, that we observed a remarkable improvement of glandular function in two patients with a disease duration of less than 3 years, possibly due to recovery of the glandular tissue. It has been reported that regeneration of glandular tissue and recovery of glandular function can occur only in patients with residual glandular function [20]. Lack of improvement in the other patients might be due to irreversible damage of the glandular tissue after a long disease duration. Improvement of salivary gland function has been reported in patients with early pSS after treatment with rituximab, an anti-CD20 monoclonal antibody. These observations emphasize the need for an earlier and more aggressive treatment in pSS patients with short disease duration [21].

Mycophenolate has been used in systemic vasculitis [22]. In the one patient with vasculitis, a reduction that has been reported previously in a pSS patient treated with MMF was observed [12]. Thus, MPA-containing compounds might be useful in this systemic manifestation.
We found a significant reduction of gamma globulins during treatment. Hypergammaglobulinemia has been proposed as a suitable target for therapy and as a primary outcome measure for the evaluation of the pSS treatment [23]. It has been suggested that reduction of B-cell hyperactivity with immunosuppressants might be the best prevention of lymphoproliferation in pSS [24]. As a low level of C4 has been associated with an increased risk of developing lymphoproliferative disease [25] and as our data show an increase of C3 and C4 levels in patients treated with MPS, this drug may be essentially beneficial in this context.

Furthermore, we found a significant reduction of $\operatorname{lgM}$ after 24 weeks, whereas no substantial reduction of $\lg G$ or $\lg A$ was detectable. In addition, a reduction of IgM-RF during the treatment was observed. Treatment with rituximab has also been accompanied by a decrease of $\operatorname{lgM}$ and IgM-RF in pSS patients without changes in $\lg G$ or $\lg A$ levels $[21,26]$.

We found no changes in anti-SSA/anti-SSB antibody titers. These antibodies are relatively stable over time in individual patients and thus are not suggested as an outcome measure of disease activity [27]. Likewise, in pSS patients receiving Bcell-depleting agents (for example, rituximab or epratuzumab), no changes in anti-SSA and anti-SSB antibodies were detected $[26,28]$.

We observed an increase of leukocytes/neutrophils during MPS treatment. Leukopenia is one of the most frequent extraglandular manifestations of the disease and is probably mediated by antineutrophil antibodies [29]. Leukopenia in pSS has been shown to be reversed by immunosuppressive treatment with corticosteroids or hydroxychloroquine [29]. Our data 
show that MPS also might be effective in treating pSS-associated leukopenia.

Patients of our cohort showed significant improvement in the general health and role emotional domains of the SF-36. A trend toward a significant increase of the mental component summary score was observed. This indicates an improvement in psychological distress, social disability due to emotional problems, and self-related health [16].

The overall tolerability of MPS in patients with pSS was acceptable. The most frequent $A E$ was mild $\mathrm{Gl}$ discomfort. Gl discomfort has been reported as the most common $A E$ leading to discontinuation of therapy in transplant recipients [6]. In SLE patients treated with MMF, Gl-related symptoms are common as well. The symptoms tend to be mild and can improve with dose reduction [8]. In transplant recipients treated with enteric-coated MPS, less severe GI AEs have been observed as compared with MMF [30].

One of our patients, after 15 days of treatment, developed pneumonia that caused hospitalization. Although the patient received only a fairly low dose of $720 \mathrm{mg}$ MPS per day for only 2 weeks, the event was possibly related to the study drug. All in all, however, compared with studies with transplant recipients [6], the incidence rate of infections was low in the present study.

\section{Conclusion}

Our findings of this open-label pilot trial in patients with pSS suggest that MPS might improve subjective glandular and extraglandular manifestations as well as some laboratory parameters. MPS promises to be an additional therapeutic option in patients with pSS, particularly in those with early disease. Controlled studies including larger numbers of patients with shorter disease durations are necessary to assess more comprehensively the efficacy and safety of MPS in pSS.

\section{Competing interests}

The authors declare that they have no competing interests.

\section{Authors' contributions}

PW participated in the data analysis and in the design of the study and drafted the manuscript. MG, HS, and HB helped with data collection, patient recruitment, and the design of the study and helped to edit the manuscript. WD helped to edit the manuscript. BS participated in the design and helped in the statistical analysis. All authors read and approved the final manuscript.

\section{Acknowledgements}

This study was financially supported, in part, by Novartis Pharma GmbH (Nürnberg, Germany).

\section{References}

1. Tomiak C, Dorner T: Sjogren's syndrome: current aspects from a rheumatological point of view. Z Rheumatol 2006, 65:505-519.

2. Allison AC, Eugui EM: Mycophenolate mofetil and its mechanisms of action. Immunopharmacology 2000, 47:85-118.

3. Hansen A, Lipsky PE, Dörner T: B cells in Sjögren's syndrome: indications for disturbed selection and differentiation in ectopic lymphoid tissue. Arthritis Res Ther 2007, 6:218.

4. Pers JO, Devauchelle V, Daridon C, Bendaoud B, Berre RL, Bordron $A$, Hutin $P$, Renaudineau $Y$, Dueymes $M$, Loisel $S$, et al: BAFF-modulated repopulation of B lymphocytes in the blood and salivary glands of Rituximab-treated patients with Sjogren's syndrome. Arthritis Rheum 2007, 56:1464-1477.

5. Placebo-controlled study of mycophenolate mofetil combined with cyclosporin and corticosteroids for prevention of acute rejection. European Mycophenolate Mofetil Cooperative Study Group. Lancet 1995, 345:1321-1325.

6. Pietruck F, Abbud-Filho M, Vathsala A, Massari PU, Po-Huang L, Nashan B: Conversion from mycophenolate mofetil to entericcoated mycophenolate sodium in stable maintenance renal transplant patients: pooled results from three international, multicenter studies. Transplant Proc 2007, 39:103-108.

7. Gaubitz M, Schorat A, Schotte H, Kern P, Domschke W: Mycophenolate mofetil for the treatment of systemic lupus erythematosus: an open pilot trial. Lupus 1999, 8:731-736.

8. Ginzler EM, Dooley MA, Aranow C, Kim MY, Buyon J, Merrill JT, Petri M, Gilkeson GS, Wallace DJ, Weisman MH, et al:: Mycophenolate mofetil or intravenous cyclophosphamide for lupus nephritis. N Engl J Med 2005, 353:2219-2228.

9. Schneider-Gold C, Hartung HP, Gold R: Mycophenolate mofetil and tacrolimus: new therapeutic options in neuroimmunological diseases. Muscle Nerve 2006, 34:284-291.

10. Appel GB, Radhakrishnan J, Ginzler EM: Use of mycophenolate mofetil in autoimmune and renal diseases. Transplantation 2005, 80:S265-S271.

11. Ahmadi-Simab K, Lamprecht $P$, Nolle B, Ai M, Gross WL: Successful treatment of refractory anterior scleritis in primary Sjogren's syndrome with rituximab. Ann Rheum Dis 2005, 64:1087-1088.

12. Willeke P, Domschke W, Gaubitz M: Mycophenolate Mofetil for the treatment of primary Sjögren's Syndrome: a case report. Ann Rheum Dis 2003, 62(Suppl 1):352.

13. Vitali C, Bombardieri S, Jonsson R, Moutsopoulos HM, Alexander EL, Carsons SE, Daniels TE, Fox PC, Fox RI, Kassan SS, et al.: Classification criteria for Sjogren's syndrome: a revised version of the European criteria proposed by the American-European Consensus Group. Ann Rheum Dis 2002, 61:554-558.

14. Kallarackal GU, Ansari EA, Amos N, Martin JC, Lane C, Camilleri JP: A comparative study to assess the clinical use of Fluorescein Meniscus Time (FMT) with Tear Break up Time (TBUT) and Schirmer's tests (ST) in the diagnosis of dry eyes. Eye 2002, 16:594-600.

15. Navazesh M, Christensen CM: A comparison of whole mouth resting and stimulated salivary measurement procedures. J Dent Res 1982, 61:1158-1162.

16. Ware JE Jr, Sherbourne CD: The MOS 36-item short-form health survey (SF-36). I. Conceptual framework and item selection. Med Care 1992, 30:473-483.

17. Strombeck B, Ekdahl C, Manthorpe R, Wikstrom I, Jacobsson L: Health-related quality of life in primary Sjogren's syndrome, rheumatoid arthritis and fibromyalgia compared to normal population data using SF-36. Scand J Rheumatol 2000, 29:20-28.

18. Fries JF, Spitz P, Kraines RG, Holman HR: Measurement of patient outcome in arthritis. Arthritis Rheum 1980, 23:137-145.

19. Mavragani CP, Moutsopoulos NM, Moutsopoulos HM: The management of Sjogren's syndrome. Nat Clin Pract Rheumatol 2006, 2:252-261.

20. Pijpe J, van Imhoff GW, Vissink A, van der Wal JE, Kluin PM, Spijkervet FK, Kallenberg CG, Bootsma $\mathrm{H}$ : Changes in salivary gland immunohistology and function after rituximab monotherapy in a patient with Sjogren's syndrome and associated MALT lymphoma. Ann Rheum Dis 2005, 64:958-960.

21. Pijpe J, van Imhoff GW, Spijkervet FK, Roodenburg JL, Wolbink GJ, Mansour K, Vissink A, Kallenberg CG, Bootsma H: Rituximab 
treatment in patients with primary Sjogren's syndrome: an open-label phase II study. Arthritis Rheum 2005, 52:2740-2750.

22. laccarino L, Rampudda M, Canova M, Della Libera S, Doria A: Mycophenolate mofetil: what is its place in the treatment of autoimmune rheumatic diseases? Autoimmun Rev 190 2007, 6:190-195.

23. Asmussen $\mathrm{KH}$, Bowman SJ: Outcome measures in Sjogren's syndrome. Rheumatology (Oxford) 2001, 40:1085-1088.

24. Mariette X: Lymphomas complicating Sjogren's syndrome and hepatitis $C$ virus infection may share a common pathogenesis: chronic stimulation of rheumatoid factor B cells. Ann Rheum Dis 2001, 60:1007-1010.

25. Theander E, Henriksson G, Ljungberg O, Mandl T, Manthorpe R, Jacobsson LT: Lymphoma and other malignancies in primary Sjogren's syndrome: a cohort study on cancer incidence and lymphoma predictors. Ann Rheum Dis 2006, 65:796-803.

26. Devauchelle-Pensec V, Pennec $Y$, Morvan J, Pers JO, Daridon $C$, Jousse-Joulin S, Roudaut A, Jamin C, Renaudineau $Y$, Roue IQ, et al.: Improvement of Sjogren's syndrome after two infusions of rituximab (anti-CD20). Arthritis Rheum 2007, 57:310-317.

27. Bowman SJ, Pillemer S, Jonsson R, Asmussen K, Vitali C, Manthorpe R, Sutcliffe N: Revisiting Sjogren's syndrome in the new millennium: perspectives on assessment and outcome measures. Report of a workshop held on 23 March 2000 at Oxford, UK. Rheumatology (Oxford) 2001, 40:1180-1188.

28. Steinfeld SD, Tant L, Burmester GR, Teoh NK, Wegener WA Goldenberg DM, Pradier O: Epratuzumab (humanised antiCD22 antibody) in primary Sjogren's syndrome: an open-label phase I/II study. Arthritis Res Ther 2006, 8:R129.

29. Schattner A, Friedman J, Klepfish A, Berrebi A: Immune cytopenias as the presenting finding in primary Sjogren's syndrome. QJM 2000, 93:825-829.

30. Granger DK: Enteric-coated mycophenolate sodium: results of two pivotal global multicenter trials. Transplant Proc 2001, 33:3241-3244. 